\title{
Hereditary Fructose Intolerance Diagnosed in Adulthood
}

\author{
Min Soo Kim¹, Jin Soo Moon', Man Jin Kim²,3, Moon-Woo Seong², Sung Sup Park², and Jae Sung Ko \\ ${ }^{1}$ Department of Pediatrics, Seoul National University College of Medicine, ${ }^{2}$ Department of Laboratory Medicine and ${ }^{3}$ Rare Disease \\ Center, Seoul National University Hospital, Seoul National University College of Medicine, Seoul, Korea
}

\author{
Article Info \\ Received June 16, 2020 \\ Revised July 22, 2020 \\ Accepted July 28, 2020 \\ Published online October 8, 2020

Corresponding Author
Jae Sung Ko
ORCID https://orcid.org/0000-0002-3064-2974
E-mail kojs@snu.ac.kr

Hereditary fructose intolerance ( $\mathrm{HFI})$ is an autosomal recessive disorder caused by a mutation in the aldolase B gene. HFI patients exhibit nausea, vomiting, abdominal pain, hypoglycemia, and elevated liver enzymes after dietary fructose exposure. Chronic exposure might lead to failure to thrive, liver failure, renal failure, and, eventually, death. HFI usually manifests in infants when they are being weaned off of breastmilk. Because HFI has an excellent prognosis when patients maintain a strict restrictive diet, some patients remain undiagnosed due to the voluntary avoidance of sweet foods. In the past, HFI was diagnosed using a fructose tolerance test, liver enzyme assays or intestinal biopsy specimens. Currently, HFI is diagnosed through the analysis of aldolase B mutations. Here, HFI was diagnosed in a 41-year-old woman who complained of sweating, nausea, and vomiting after consuming sweets. She had a compound heterozygous mutation in the aldolase B gene; gene analysis revealed pathogenic nonsense (c.178C >T, p.Arg60Ter) and frameshift (c.360_363delCAAA, p.Asn120LysfsTer32) variants. This is the first report of a Korean HFI patient diagnosed in adulthood. (Gut Liver 2021;15:142-145)

Key Words: Fructose intolerance; Aldolase B

\section{INTRODUCTION}

Hereditary fructose intolerance (HFI; OMIM\# 229600) is a rare autosomal recessive disorder caused by a deficiency in aldolase B, usually diagnosed in childhood. ${ }^{1,2}$ After intake, fructose is phosphorylated to fructose 1-phosphate (F 1-P). F 1-P is then catabolized by aldolase $\mathrm{B}^{3}$ In HFI patients, F 1-P accumulates and inhibits gluconeogenesis and glycogenolysis. Early manifestations of fructose intake include nausea, vomiting, abdominal pain, hypoglycemia, elevated liver enzymes, and faintness. Chronic exposure to fructose might cause failure to thrive, liver failure, renal failure, and, eventually, death. ${ }^{4}$ Severity depends on the timing and amount of fructose intake. Patients usually show their first symptoms in the infantile period when exposed to fructose and sucrose-containing foods. ${ }^{1}$ Recurrent symptoms make diagnosis possible in childhood; however, some remain undiagnosed until adulthood. ${ }^{5,6}$ Traditionally, HFI was diagnosed using a fructose tolerance test or an enzyme assay of liver or small intestine biopsy. Nowadays, diagnosis occurs through aldolase B gene analysis. Here we report a 41-year-old woman diagnosed with HFI in adulthood through gene analysis. This is the first report of a Korean HFI patient diagnosed in adulthood.

This study was approved by the Institutional Review Board of Seoul National University Hospital (IRB number: 2002-132-1104). Informed consent was obtained from the patient.

\section{CASE REPORT}

A 41-year-old woman visited an outpatient clinic due to repeated nausea and vomiting after the administration of fruits, sucrose, or fructose-containing foods. A lifelong history of aversion to sweets was revealed. She was breastfed until 12 months of age. Her mother tried giving her fruits, but she refused them. After being forced to eat fruits at the age of three, she showed symptoms of nausea, vomiting, and visual disturbance. The patient then continuously avoided sweets. In adulthood, after one sip of a beverage, nausea, vomiting, and diarrhea occurred. Two hours later, 
she presented with a cold sweat and faintness. She had no family history of liver or genetic disease. Other family members were asymptomatic. Her height was $160 \mathrm{~cm}$ (50th percentile), and her weight was $50.2 \mathrm{~kg}$ (25th to 50 th percentile). Her physical examination was normal; no hepatomegaly or splenomegaly was found. Laboratory findings showed no abnormality as well. Her white blood cell count was $8,460 / \mathrm{mm}^{3}$ (neutrophil $64 \%$, lymphocytes $30.5 \%$, monocyte $4.8 \%$, eosinophil $0.5 \%$, and basophil $0.2 \%$ ), hemoglobin $14.1 \mathrm{~g} / \mathrm{dL}$, platelet $261,000 / \mathrm{mm}^{3}$, calcium 8.8 $\mathrm{mg} / \mathrm{dL}$, phosphorus $3.5 \mathrm{mg} / \mathrm{dL}$, glucose $96 \mathrm{mg} / \mathrm{dL}$, uric acid $3.0 \mathrm{mg} / \mathrm{dL}$, total cholesterol $180 \mathrm{mg} / \mathrm{dL}$, total protein $7.5 \mathrm{~g} / \mathrm{dL}$, albumin $4.5 \mathrm{~g} / \mathrm{dL}$, total bilirubin $0.7 \mathrm{mg} / \mathrm{dL}$, alkaline phosphatase $61 \mathrm{IU} / \mathrm{L}$, aspartate aminotransferase 19 IU/L, alanine aminotransferase 16 IU/L, blood urea nitrogen $12 \mathrm{mg} / \mathrm{dL}$, creatinine $0.49 \mathrm{mg} / \mathrm{dL}$, prothrombin time 0.97 INR (international normalized ratio), activated partial thrombin time 30.1 seconds, and no urinalysis abnormality. Liver ultrasonography showed normal size, shape, and echotexture without focal lesions.

Through her history, HFI was suspected, and gene analysis of aldolase B was completed. Genomic DNA was extracted, and the Agilent SureSelectXT Human all Exon 50 $\mathrm{Mb}$ kit was used to target the exon regions. These targeted regions were sequenced using the Illumina HiSeq sequencing system with 100 bp paired-end reads. All sequence variants were confirmed by Sanger sequence analysis. The patient showed known heterozygous pathogenic nonsense (c.178C $>$ T, p.Arg60Ter) variant and known pathogenic frameshift (c.360_363delCAAA, p.Asn120LysfsTer32) variant. ${ }^{7,8}$ She began a fructose-restricted diet after counseling with a dietician.

\section{DISCUSSION}

HFI has an estimated incidence of 1:18,000 to $1: 31,000 .^{9-11}$ HFI is caused by a mutation in aldolase $B$, which results in the accumulation of F 1-P. ${ }^{12}$ HFI often manifests in young infants when fructose or sucrose-containing foods are first introduced after breastfeeding. Symptoms usually manifest as nausea, vomiting, and aversion to fructose-containing foods. Prolonged fructose ingestion may cause liver and renal failure; physical examination might reveal hepatomegaly and jaundice; the main consequence is depletion of phosphate due to the phosphorylation of fructose. As a result, patients show hypophosphatemia, hyperuricemia, hypermagnesemia, hypoglycemia, and acidosis after fructose loading. Lack of phosphate interrupts all cellular processes requiring phosphorylation or adenosine triphosphate, including glycogenolysis and gluconeogenesis. This explains why the administration of glucagon does not correct hypoglycemia (Fig. 1).

Patients voluntarily avoid sweet foods. When fructose is restricted after the infantile period, HFI may remain undiagnosed until adulthood. In the 1970s, undiagnosed HFI patients were exposed to the danger of using fructose or sorbitol as a source of parenteral nutrition; several fatal cases were reported. ${ }^{13}$ Fructose and sorbitol infusions are no longer used. Nowadays, glucose and lipid solutions are preferred sources of energy.

In this case, the patient refused sweet foods since infanthood. Early exclusion of sucrose or fructose in the diet improves symptoms and prevents further sequelae. The patient had no comorbidity or underlying disease; laboratory findings were normal. Physical exam showed no hepatomegaly or other abnormal findings. Although this patient was referred for unpleasant symptoms after eating sweet foods, patients may present with acute liver failure or severe nausea and vomiting. HFI should be considered

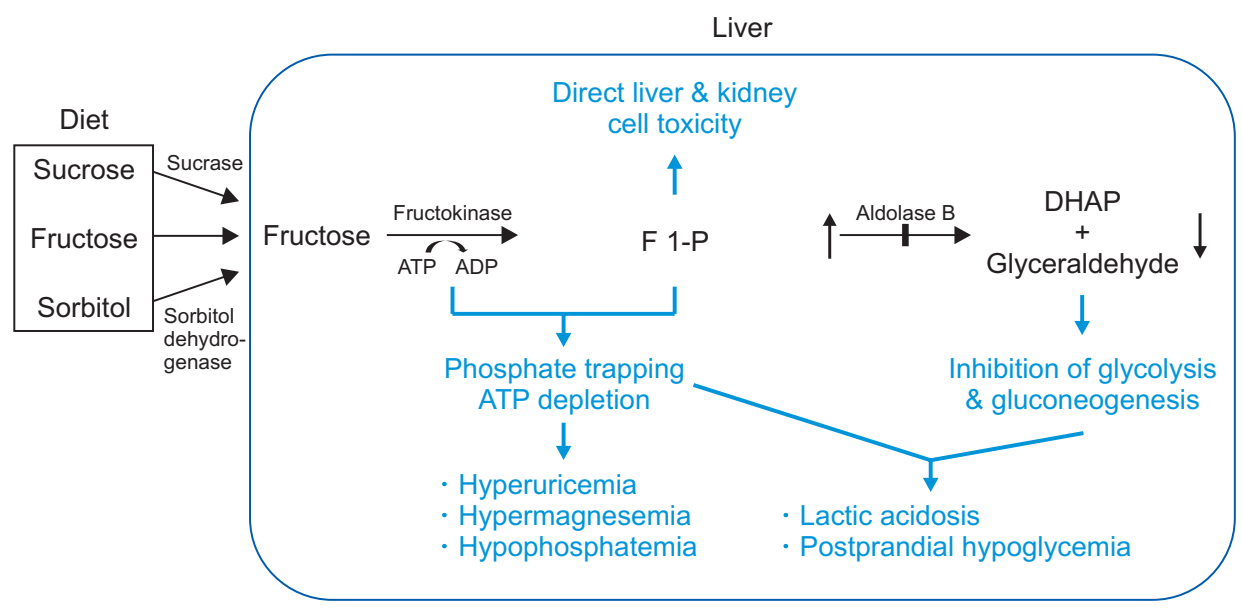

Fig. 1. Pathophysiology of hereditary fructose intolerance (HFI). Deficiency of aldolase B leads to the accumulation of fructose 1-phosphate (F 1-P), which causes direct toxicity to the liver and kidney. Glycolysis and gluconeogenesis are downregulated due to the inhibition of DHAP and glyceraldehyde, which enters the pathway. Moreover, ATP is depleted as phosphorylation continues, blocking all processes requiring ATP.

ATP, adenosine triphosphate; ADP, adenosine diphosphate; DHAP, dihydroxyacetone phosphate. 
as a possible diagnosis in a patient showing unpleasant symptoms after sugar loading. Symptoms may vary depending on the age of exposure and intensity of fructose intake, possibly as severe as acute liver failure. Cases of acute liver failure in neonates were reported when exposed to sucrose-containing formula; ${ }^{14}$ recurrent episodes of hepatitis have been reported in infancy. ${ }^{15}$ In adults, patients show a lifelong history of avoiding sweet fruits and nausea after small amounts of sweets. ${ }^{5,6}$ Therefore, thorough history taking is critical for HFI diagnosis. Formerly HFI was diagnosed through activity assay of aldolase B in tissue biopsy specimens or fructose tolerance test. Biopsy techniques have risks of pain, bleeding, and complications of sedation. Fructose tolerance tests induce acute symptoms of hypoglycemia, nausea, and vomiting. These invasive tests are unnecessary since genetic testing for HFI is safer, and should be frequently and promptly performed.

There have been two HFI Korean childhood cases; this is the first report of a Korean adult patient. ${ }^{15,16}$ One case was diagnosed through an enzyme assay of an intestinal and liver biopsy in 2002. The other case was diagnosed through genetic testing, which showed a c.758_759insT (p.V253fsX24) homozygote in 2012. More than 50 mutations are known to cause HFI, but frequencies of mutant alleles in East Asia are not well known. c.448G>C (p.Ala150Pro) is the most common mutation worldwide, other common mutations are c.524C $>\mathrm{A}$ (p.Ala175Asp), c. $1005 \mathrm{C}>\mathrm{G}$ (p.Asn335Lys), c.360_363delCAAA (p.Asn120LysfsTer32), and c.178C $>$ T (p.Arg60Ter) ${ }^{10,11,17-19}$

Interestingly, there is no report on the p.Ala150Pro mutation in Asian HFI patients. In Asia, three HFI cases diagnosed with genetic testing were reported. Besides one Korean report of novel frameshift mutation, the other two cases were reported in Japan and China, respectively. The Japanese patient was homozygote for a nonsense mutation c.720C >A (p.Cys240Ter) from consanguineous parents. ${ }^{20}$ The Chinese patient, whose parents were cousins, was homozygote for a frameshift mutation c.479_482delAACA. ${ }^{21}$ These mutations are uncommon in Western countries. In Asia, few HFI cases were reported, genetic testing was rare, and no study was conducted on the frequency of HFI. Two mutations found in our patient, c.360_363delCAAA and c. $178 \mathrm{C}>\mathrm{T}$, are the third and fourth most common HFI mutations in America, respectively, and are also widespread in Europe. $^{17}$

c.360_363delCAAA and c.178C $>$ T mutations both create premature stop codons, resulting in a truncated protein with deteriorated function. Frameshift and nonsense mutations cause more severe changes in protein structure than missense mutations. However, recent studies show that phenotypes do not differ from genotypes. Several patients

are reported to have two null alleles without an exhibition of severe symptoms or unusual phenotypes. ${ }^{10,17,21}$ In this case, the patient also showed typical symptoms of classic HFI and excellent prognosis after fructose restriction. It implies that harboring two null alleles does not lead to a severe phenotype.

After the diagnosis of HFI has been made, an expert dietician should develop a strict restrictive diet. Careful advice on medication should also be provided since sucrose and sorbitol are common components in syrups and tablets. Recently, higher intrahepatic triglyceride content was reported in HFI patients than healthy control groups, even with a fructose-restricted diet. ${ }^{22}$ Nonalcoholic fatty liver disease is also a potential threat in HFI; regular follow-up is needed. Prognosis is excellent if patients maintain a strict exclusion diet. Therefore, repeated education is essential for HFI patients.

HFI is a rare, unfamiliar, and underdiagnosed disorder in Korea. HFI should be considered in individuals with characteristic discomforts and clinical manifestations following exposure to fructose, sucrose, or sorbitol. Knowing the symptoms and pathophysiology of HFI could alter the clinical course of HFI patients. Simply restricting fructose could dramatically change the patient's outcome. Therefore, we report the first adult case of a Korean HFI patient to facilitate early recognition and treatment. Further studies will provide opportunities to determine the incidence of HFI in the Korean population.

\section{CONFLICTS OF INTEREST}

No potential conflict of interest relevant to this article was reported.

\section{AUTHOR CONTRIBUTIONS}

Data analysis and interpretation: M.J.K., M.W.S., S.S.P. Data acquisition, drafting of the manuscript, study concept and design: M.S.K. Administrative, technical, and material support: J.S.M. Study concept and design, critical revision of the manuscript for important intellectual content, and study supervision: J.S.K.

\section{ORCID}

Min Soo Kim

Jin Soo Moon

Man Jin Kim https://orcid.org/0000-0001-7244-2359 https://orcid.org/0000-0001-9760-297X

https://orcid.org/0000-0002-9345-6976 
Moon-Woo Seong

https://orcid.org/0000-0003-2954-3677

Sung Sup Park https://orcid.org/0000-0003-3754-4848

Jae Sung Ko

\section{REFERENCES}

1. Ali M, Rellos P, Cox TM. Hereditary fructose intolerance. J Med Genet 1998;35:353-365.

2. Odièvre M, Gentil C, Gautier M, Alagille D. Hereditary fructose intolerance in childhood: diagnosis, management, and course in 55 patients. Am J Dis Child 1978;132:605-608.

3. Bouteldja N, Timson DJ. The biochemical basis of hereditary fructose intolerance. J Inherit Metab Dis 2010;33:105-112.

4. Mock DM, Perman JA, Thaler M, Morris RC Jr. Chronic fructose intoxication after infancy in children with hereditary fructose intolerance: a cause of growth retardation. $\mathrm{N}$ Engl J Med 1983;309:764-770.

5. Yasawy MI, Folsch UR, Schmidt WE, Schwend M. Adult hereditary fructose intolerance. World J Gastroenterol 2009; 15:2412-2413.

6. Burmeister LA, Valdivia T, Nuttall FQ. Adult hereditary fructose intolerance. Arch Intern Med 1991;151:773-776.

7. National Center for Biotechnology Information (NCBI). ClinVar: NM_000035.4(ALDOB):c.178C > T [Internet]. Bethesda: NCBI; c2020 [cited 2020 May 14]. Available from: https://www.ncbi.nlm.nih.gov/clinvar/variation/ VCV000000472.4.

8. National Center for Biotechnology Information (NCBI). ClinVar: NM_000035.4(ALDOB):c.356_359CAAA [Internet]. Bethesda: NCBI; c2020 [cited 2020 May 14]. Available from: https://www.ncbi.nlm.nih.gov/clinvar/variation/ VCV000188861.6.

9. James CL, Rellos P, Ali M, Heeley AF, Cox TM. Neonatal screening for hereditary fructose intolerance: frequency of the most common mutant aldolase B allele (A149P) in the British population. J Med Genet 1996;33:837-841.

10. Santer R, Rischewski J, von Weihe M, et al. The spectrum of aldolase B (ALDOB) mutations and the prevalence of hereditary fructose intolerance in Central Europe. Hum Mutat 2005;25:594.

11. Gruchota J, Pronicka E, Korniszewski L, et al. Aldolase B mutations and prevalence of hereditary fructose intolerance in a Polish population. Mol Genet Metab 2006;87:376-378.

12. Oberhaensli RD, Rajagopalan B, Taylor DJ, et al. Study of hereditary fructose intolerance by use of $31 \mathrm{P}$ magnetic resonance spectroscopy. Lancet 1987;2:931-934.

13. Schulte MJ, Lenz W. Fatal sorbitol infusion in patient with fructose-sorbitol intolerance. Lancet 1977;2:188.

14. Li H, Byers HM, Diaz-Kuan A, et al. Acute liver failure in neonates with undiagnosed hereditary fructose intolerance due to exposure from widely available infant formulas. Mol Genet Metab 2018;123:428-432.

15. Choi HW, Lee YJ, Oh SH, et al. A novel frameshift mutation of the ALDOB gene in a Korean girl presenting with recurrent hepatitis diagnosed as hereditary fructose intolerance. Gut Liver 2012;6: 126-128.

16. Kang EK, Yang HR, Seo JK, et al. A case of hereditary fructose intolerance. J Korean Pediatr Soc 2002;45:120-124.

17. Coffee EM, Yerkes L, Ewen EP, Zee T, Tolan DR. Increased prevalence of mutant null alleles that cause hereditary fructose intolerance in the American population. J Inherit Metab Dis 2010;33:33-42.

18. Cross NC, de Franchis R, Sebastio G, et al. Molecular analysis of aldolase B genes in hereditary fructose intolerance. Lancet 1990; 335:306-309.

19. Davit-Spraul A, Costa C, Zater M, et al. Hereditary fructose intolerance: frequency and spectrum mutations of the aldolase $B$ gene in a large patients cohort from France: identification of eight new mutations. Mol Genet Metab 2008;94:443447.

20. Kajihara S, Mukai T, Arai Y, Owada M, Kitagawa T, Hori K. Hereditary fructose intolerance caused by a nonsense mutation of the aldolase B gene. Am J Hum Genet 1990;47:562567.

21. Chi ZN, Hong J, Yang J, et al. Clinical and genetic analysis for a Chinese family with hereditary fructose intolerance. Endocrine 2007;32:122-126.

22. Simons N, Debray FG, Schaper NC, et al. Patients with aldolase $\mathrm{b}$ deficiency are characterized by increased intrahepatic triglyceride content. J Clin Endocrinol Metab 2019;104:5056-5064. 